\title{
Bounded-degree graphs can have arbitrarily large slope numbers
}

\author{
János Pach* and Dömötör Pálvölgyi \\ Rényi Institute, Hungarian Academy of Sciences \\ Submitted: Oct 21, 2005; Accepted: Dec 22, 2005; Published: Jan 7, 2006 \\ Mathematics Subject Classification: 05C62
}

\begin{abstract}
We construct graphs with $n$ vertices of maximum degree 5 whose every straight-line drawing in the plane uses edges of at least $n^{1 / 6-o(1)}$ distinct slopes.
\end{abstract}

A straight-line drawing of a graph $G=(V(G), E(G))$ is a layout of $G$ in the plane such that the vertices are represented by distinct points, the edges are represented by (possibly crossing) line segments connecting the corresponding point pairs and not passing through any other point that represents a vertex. If it creates no confusion, the vertex (edge) of $G$ and the point (segment) representing it will be denoted by the same symbol. Wade and $\mathrm{Chu}$ [WC94] defined the slope number $\operatorname{sl}(G)$ of $G$ as the smallest number of distinct edge slopes used in a straight-line drawing of $G$. Dujmović et al. [DSW04] asked whether the slope number of a graph of maximum degree $d$ can be arbitrarily large. The following short argument shows that the answer is yes for $d \geq 5$.

Define a "frame" graph $F$ on the vertex set $\{1, \ldots, n\}$ by connecting vertex 1 to 2 by an edge and connecting every $i>2$ to $i-1$ and $i-2$. Adding a perfect matching $M$ between these $n$ points, we obtain a graph $G_{M}:=F \cup M$. The number of different matchings is at least $(n / 3)^{n / 2}$. Let $G$ denote the huge graph obtained by taking the union of disjoint copies of all $G_{M}$. Clearly, the maximum degree of the vertices of $G$ is five. Suppose that $G$ can be drawn using at most $S$ slopes, and fix such a drawing.

For every edge $i j \in M$, label the points in $G_{M}$ corresponding to $i$ and $j$ by the slope of $i j$ in the drawing. Furthermore, label each frame edge $i j(|i-j| \leq 2)$ by its slope. Notice that no two components of $G$ receive the same labeling. Indeed, up to translation and scaling, the labeling of the edges uniquely determines the positions of the points representing the vertices of $G_{M}$. Then the labeling of the vertices uniquely determines the edges belonging to $M$. Therefore, the number of different possible labelings, which is

\footnotetext{
${ }^{*}$ Supported by NSF grant CCR-0514079 and grants from NSA, PSC-CUNY, Hungarian Research Foundation, and BSF
} 
$S^{|F|+n}<S^{3 n}$, is an upper bound for the number of components of $G$. On the other hand, we have seen that the number of components (matchings) is at least $(n / 3)^{n / 2}$. Thus, for any $S$ we obtain a contradiction, provided that $n$ is sufficiently large.

With some extra care one can refine this argument to obtain

Theorem. For any $d \geq 5$, there exist graphs $G$ with $n$ vertices of maximum degree $d$, whose slope numbers satisfy $\operatorname{sl}(G) \geq n^{\frac{1}{2}-\frac{1}{d-2}-o(1)}$.

Proof. Now instead of a matching, we add to the frame $F$ in every possible way a $(d-4)$ regular graph $R$ on the vertex set $\{1, \ldots, n\}$. Thus, we obtain at least $(c n / d)^{(d-4) n / 2}$ different graphs $G_{R}:=F \cup R$, each having maximum degree at most $d$ (here $c>0$ is a constant; see e.g. [BC78]). Suppose that each $G_{R}$ can be drawn using $S$ slopes $\sigma_{1}<\ldots<\sigma_{S}$. Now we cannot insist that these slopes are the same for all $G_{R}$, therefore, these numbers will be regarded as variables.

Fix a graph $G_{R}=F \cup R$ and one of its drawings with the above properties, in which vertex 1 is mapped into the origin and vertex 2 is mapped into a point whose $x$-coordinate is 1 . Label every edge belonging to $F$ by the symbol $\sigma_{k}$ representing its slope. Furthermore, label each vertex $j$ with a $(d-4)$-tuple of the $\sigma_{k}$ s: with the symbols corresponding to the slopes of the $d-4$ edges incident to $j$ in $R$ (with possible repetition). Clearly, the total number of possible labelings of the frame edges and vertices is at most $S^{|F|+(d-4) n}<S^{(d-2) n}$. Now the labeling itself does not necessarily identify the graph $G_{R}$, because we do not know the actual values of the slopes $\sigma_{k}$.

However, we can show that the number of different $G_{R}$ s that receive the same labeling cannot be too large. To prove this, first notice that for a fixed labeling of the edges of the frame, the coordinates of every vertex $i$ can be expressed as the ratio of two polynomials of degree at most $n$ in the variables $\sigma_{1}, \ldots, \sigma_{S}$. Indeed, let $\sigma(i j)$ denote the label of $i j \in F$, and let $x(i)$ and $y(i)$ denote the coordinates of vertex $i$. Since, by assumption, we have $x(1)=y(1)=0$ and $x(2)=1$, we can conclude that $y(2)=\sigma(12)$. We have the following equations for the coordinates of 3 :

$$
y(3)-y(1)=\sigma(13)(x(3)-x(1)), \quad y(3)-y(2)=\sigma(23)(x(3)-x(2)) .
$$

Solving them, we obtain

$$
x(3)=\frac{\sigma(12)-\sigma(23)}{\sigma(13)-\sigma(23)}, \quad y(3)=\frac{\sigma(13)(\sigma(12)-\sigma(23))}{\sigma(13)-\sigma(23)},
$$

and so on. In particular, $x(i)=\frac{Q_{i}\left(\sigma_{1}, \ldots, \sigma_{S}\right)}{Q_{i}^{\prime}\left(\sigma_{1}, \ldots, \sigma_{S}\right)}$, for suitable polynomials $Q_{i}$ and $Q_{i}^{\prime}$ of degree at most $i-1$. Moreover, $Q_{j}^{\prime}$ is a multiple of $Q_{i}^{\prime}$ for all $j>i$.

Since

$$
x(i)-x(j)=\frac{Q_{i} \frac{Q_{j}^{\prime}}{Q_{i}^{\prime}}-Q_{j}}{Q_{j}^{\prime}},
$$

we can decide whether the image of $i$ is to the left of the image of $j>i$, to the right of it, or they have the same $x$-coordinate, provided that we know the "sign pattern" of the 
polynomials $P_{i j}^{\prime}:=Q_{i} \frac{Q_{j}^{\prime}}{Q_{i}^{\prime}}-Q_{j}$ and $Q_{j}^{\prime}$, i.e., we know which of them are positive, negative, or zero.

Now if we also know that $\sigma_{k}$ is one of the labels associated with vertex $i$, the condition that the line connecting $i$ and $j$ has slope $\sigma_{k}$ can be rewritten as

$$
\frac{y(i)-y(j)}{x(i)-x(j)}-\sigma_{k}=\frac{\sigma(1 i) Q_{i} Q_{j}^{\prime}-\sigma(1 j) Q_{i}^{\prime} Q_{j}}{Q_{i} Q_{j}^{\prime}-Q_{i}^{\prime} Q_{j}}-\sigma_{k}=0,
$$

that is, as a polynomial equation $P_{i j k}\left(\sigma_{1}, \ldots, \sigma_{S}\right)=0$ of degree at most $2 n$. For a fixed labeling of the frame edges and vertices, there are $d-4$ labels $k$ associated with a vertex $i$, so that the number of these polynomials $P_{i j k}$ is at most $(d-4) n(n-1)$. Thus, together with the $\left(\begin{array}{l}n \\ 2\end{array}\right)+n$ polynomials $P_{i j}^{\prime}$ and $Q_{j}^{\prime}$, we have fewer than $d n^{2}$ polynomials, each of degree at most $2 n$.

It is easy to verify that, for any fixed labeling, the sign pattern of these polynomials uniquely determines the graph $G_{R}$. (Observe that if the label of a vertex $i$ is a $(d-4)$-tuple containing the symbol $\sigma_{k}$, then from the sign pattern of the above polynomials we can reconstruct the sequence of all vertices that belong to the line of slope $\sigma_{k}$ passing through $i$, from left to right. From this sequence, we can select all elements whose label contains $\sigma_{k}$, and determine all edges of $R$ along this line.)

To conclude the proof, we need the Thom-Milnor theorem [BPR03]: Given $N$ polynomials in $S \leq N$ variables, each of degree at most $2 n$, the number of sign patterns determined by them is at most $(C N n / S)^{S}$, for a suitable constant $C>0$.

In our case, the number of graphs $G_{R}$ is at most the number of labelings $\left(<S^{(d-2) n}\right)$ multiplied by the maximum number of sign patterns of the above $<d n^{2}$ polynomials of degree at most $2 n$. By the Thom-Milnor theorem, this latter quantity is smaller than $\left(C d n^{3}\right)^{S}$. Thus, the number of $G_{R}$ is at most $S^{(d-2) n}\left(C d n^{3}\right)^{S}$. Comparing this to the lower bound $(\mathrm{cn} / \mathrm{d})^{(d-4) n / 2}$ stated in the first paragraph of the proof, we obtain that $S \geq n^{\frac{1}{2}-\frac{1}{d-2}-o(1)}$, as required.

Acknowledgment. Barát et al. [BMW05] independently found some similar, but slightly weaker results for the slope number. In particular, for $d=5$, they have a more complicated proof for the existence of graphs with maximum degree five and arbitrarily large slope numbers, that does not give any good explicit lower bound for the growth rate of the slope number, as the number of vertices tends to infinity. They have also established similar results for the geometric thickness, defined as the smallest integer $S$ with the property that the graph $G$ admits a straight-line drawing, in which the edges can be colored by $S$ colors so that no two edges of the same color cross each other [E04]. Clearly, this number cannot exceed $\operatorname{sl}(G)$.

We are grateful to B. Aronov for his valuable remarks. 


\section{References}

[BMW05] J. Barát, J. Matoušek, and D.R. Wood: Bounded-degree graphs have arbitrarily large geometric thickness, The Electronic J. Combin., 13 (2006), R3.

[BPR03] S. Basu, R. Pollack, and M.-F. Roy: Algorithms in Real Algebraic Geometry, Springer-Verlag, Berlin, 2003.

[BC78] E.A. Bender and E.R. Canfield: The asymptotic number of labeled graphs with given degree sequences, J. Combin. Theory Ser. A 24 (1978), 296-307.

[DSW04] V. Dujmović, M. Suderman, and D.R. Wood: Really straight graph drawings, in: Graph Drawing (GD '04) (J. Pach, ed.), Lecture Notes in Computer Science 3383, Springer-Verlag, Berlin, 2004, 122-132.

[E04] D. Eppstein: Separating thickness from geometric thickness, in: Towards a Theory of Geometric Graphs (J. Pach, ed.), Contemporary Mathematics 342, Amer. Math. Soc., 2004, 75-86.

[WC94] G.A. Wade and J.-H. Chu: Drawability of complete graphs using a minimal slope set, The Computer J. 37/2 (1994), 139-142. 